"This is the peer reviewed version of the following article: [Nursing philosophy : an international journal for healthcare professionals, 2020, pp. e12320] which has been published in final form at [https://onlinelibrary.wiley.com/doi/abs/10.1111/nup.12320 [I purposes in accordance with Wiley Terms and Conditions for Self-Archiving." 


\title{
Bowen Family Systems Theory: mapping a framework to support critical care nurses' wellbeing and care quality
}

\begin{abstract}
Intensive care nursing is prone to episodic anxiety linked to patients' immediate needs for treatment. Balancing biomedical interventions with compassionate patient-centred nursing can be particularly anxiety provoking. These patterns of anxiety may impact compassion and patient-centred nursing.
\end{abstract}

The aim of this paper is to discuss the application of Bowen Family Systems Theory to intensive care nursing, mapping a framework to support critical care nurses' wellbeing and, consequently, the quality of care they provide. This article is founded on research, theoretical papers and texts focussed on Bowen Family Systems Theory (BFST), and findings from a constructivist study on patient-centred nursing and compassion in the intensive care unit.

The goal of Bowen Family Systems Theory is to empower individuals, decreasing blame and reactivity. Bowen Family Systems Theory can be applied to the sometimes intimate relationships that develop in this environment, aiding understanding of nurses' experience of compassion satisfaction and fatigue. Where organisational factors and management styles fall short in supporting critical care nurses to meet expectations, BFST can offer a perspective on the processes that occur within the intensive care unit, impacting nurse wellbeing and quality of care. This paper makes plain the importance of understanding the anxiety that occurs within the intensive care unit as a system, so that individuals, such as critical care nurses, can be supported appropriately to ensure nurse wellbeing and quality care. 


\section{Keywords (up to 10)}

Compassion, critical care, differentiation, family systems theory, intensive care unit, nurses, patient-centred, workplace culture

\section{INTRODUCTION}

The goal of healthcare professionals working in intensive care is to use their skills, knowledge and technology to maintain life and alleviate suffering, and many experience a sense of failure if their patient dies (Beckstrand, Hadley, Luthy, \& Macintosh, 2017). The intensive care unit epitomises the medical ideology of cure and restoration of health for all. However, death in the intensive care unit is a stark and not infrequent reality, no matter how much effort is made to avoid this. In this and other situations, where the expectations of patients, families, health professionals and the healthcare service as a whole are unmet, an uncomfortable work environment can result for nurses and their colleagues (Jakimowicz, Perry, \& Lewis, 2017b)

Critical care nurses are generally expected to provide nursing that is patient-centred; this model of nursing is emphasised in health service core values internationally (WHO, 2014). Patientcentred nursing (McCormack \& McCance, 2016) and Patient and Family Centred Care (PFCC) (Mitchell et al., 2016) are models of care now recognised as capable of meeting the expectations and needs of both contemporary health services and the community. However, the expectation that critical care nurses will deliver compassionate patient-centred nursing (Jakimowicz \& Perry, 2015) may be difficult to meet as they balance the immediate biomedical needs of their patient with providing a compassionate presence (Kelleher, 2006).

Compassion is a gauge of care quality that is not always achieved in healthcare (Francis, 2013). Compassion has been defined as an emotive response to witnessing the distress or suffering of 
an individual and the counter response driving the need to relieve their suffering (Figley, 1995). Compassion satisfaction is felt when critical care nurses are able to relieve their patients' suffering: the positive aspect of caring. Death rates are high in ICU (Beckstrand et al., 2017) and in this cure-focussed setting (Kelleher, 2006), compassion fatigue can result from the sense of failure that critical care nurses (and other members of the team) may feel when their patient dies despite their best efforts (Epp, 2012). Compassion fatigue is comprised of burnout and secondary traumatic stress and is described as resulting from the effort of caring for those who are suffering (Figley, 1995); it may occur throughout a nurse's career, and impacts their professional quality of life.

Delivery of direct patient care is a core aspect of critical care nurses' role. Critical care nurses are required to make numerous decisions while simultaneously working with advanced technology and undertaking complex activities, all in a high pressure environment (Abbey, Chaboyer, \& Mitchell, 2012). Critical care nurses are exposed to extremely stressful and unpredictable situations and are vulnerable to fatigue (Jones, Winch, Strube, Mitchell, \& Henderson, 2016) and stress-related consequences (Karanikola et al., 2014). Critical care nurses rely on each other for emotional and practical support, encouraging and motivating each other in their challenging roles (Jakimowicz, Perry, \& Lewis, 2017c). Relationships within critical care have been reported as complex (Jakimowicz et al., 2017c) with the structure and culture of the unit playing an important role in delivery of professional and compassionate nursing care (Jones et al., 2016). Poor collaboration between nurses, physicians, colleagues and/or the management team can lead to nurses' moral distress and intention to resign (Karanikola et al., 2014), and can impact delivery of effective patient-centred nursing (Jakimowicz, Perry, \& Lewis, 2017a). Therefore, it is important to examine the organisational structure and interrelationships occurring in intensive care units to better understand patterns 
of behaviour. In turn, this can help to develop recommendations to improve workplace culture, enhance collaboration, communication, workforce retention and health professional wellbeing.

Bowen Family Systems Theory (BFST) focuses on patterns within a family or group of individuals with goals of defusing anxiety and increasing individual levels of differentiation and growth, resulting in more collaborative and healthy teams (Bowen, 1978). Collaborative, collegial and inter-professional relationships (Jakimowicz et al., 2017c), work practice congruency (Jones et al., 2016), teamwork and workplace culture have been identified as factors impacting delivery of compassionate patient-centred nursing, nurse wellbeing and workforce retention in intensive care units. The objective of BFST is to empower individuals through working with groups (Kerr, 2012) such as managers and/or bedside critical care nurses, with a view to move away from a culture of blame and reactivity to a healthier environment.

BFST purpose and goals show congruence with the findings of a recent constructivist study conducted by the authors to explore patient-centred nursing and compassion in Australian intensive care units (Jakimowicz et al., 2017b). Consequently, the relevance and application of this theory for critical care nursing was examined using strategies similar to those used by Walker and Avant (2011). This method of theory derivation involves selecting a parent theory, identifying concepts within that theory and mapping it against known ideas and/or structures within a given field; in this case, intensive care nursing. Using this process alongside constructivist methods can contribute to development of a conceptual framework whereby existing knowledge can be linked with new knowledge. This approach supports examination of the concepts of Bowen Family Systems Theory mapped against findings from a study which concluded that intimate and complex relationships developed in the context of critical care. 


\section{Background}

\section{Bowen Family Systems Theory}

\section{Historical perspective}

Bowen Family Systems Theory originated in the 1950-60's by Murray Bowen, an American psychiatrist. Bowen initially researched adult patients who suffered from schizophrenia and lived with their parents, defining the field of family therapy as focussing on human interactions rather than symptomatic behaviours. He developed these ideas further into family systems theory (Kerr \& Bowen, 1988). Bowen discovered a unique relationship between mothers and their adult children leading to the initial concept of differentiation of self or separating thoughts from feelings (Bowen, 1978). He advanced the work by adding seven more concepts to develop contemporary family systems theory (Kerr, 2000). From early in its use, BFST has been applied to organisations; Bowen himself recognised anxiety within his own place of work (Kerr \& Bowen, 1988). He applied his theory to his own working situation in order to identify and alleviate anxiety for himself and his colleagues. Since then BFST has been adopted in many business structures (Chambers, 2009; Kott, 2014; Sagar \& Wiseman, 1982).

\section{Components of Bowen Family Systems Theory}

Bowen Family Systems Theory (BFST) is comprised of eight states that interconnect and outline chronic anxiety and behavioural patterns occurring within family or group relationships. The eight states of BFST, which are briefly outlined in Table 1, include differentiation of self; nuclear family emotional systems; triangles; family projection process; emotional cut-off; multi-generational transmission process; sibling position; and societal regression. 
Bowen surmises that anxiety is a source of dysfunction (Kerr \& Bowen, 1988). His theory focuses on identifying patterns that develop in systems (or families) that may cause or alleviate anxiety. Bowen (1978) suggests that the key trigger of anxiety in groups is one of perceived extreme closeness or distance within relationships; the degree of anxiety experienced within a group is underpinned by the level of stress and sensitivity to any given pattern that is handed down the generations, or transmitted from those more senior (Kerr \& Bowen, 1988). If individual members of the group react emotionally to relational dilemmas rather than intellectually examining the perceived emotional demands, a chronic state of anxiety may become part of the system's culture (Bowen, 1978).

Table 1 - Bowen Family Systems Theory concepts

\begin{tabular}{|c|c|}
\hline Bowen Theory concept & Definition \\
\hline Differentiation of self & $\begin{array}{l}\text { The ability to maintain a degree of autonomy within a group, or } \\
\text { to keep thoughts and emotions (or emotional reactions) separate, } \\
\text { especially when confronted with pressure of togetherness or peer } \\
\text { group pressure. }\end{array}$ \\
\hline Emotional Process & $\begin{array}{l}\text { The notion that all mechanisms within a group may be } \\
\text { generalised across other similar groups. }\end{array}$ \\
\hline Emotional Cut-off & $\begin{array}{l}\text { A consequence of emotional 'stuckness', or when the anxiety and } \\
\text { distress due to intense closeness becomes intolerable and the } \\
\text { individual reacts by becoming distant and unavailable } \\
\text { emotionally. }\end{array}$ \\
\hline Family Projection & $\begin{array}{l}\text { Unresolved problems of the parents (or leaders) are projected } \\
\text { onto the children (or organisation members). For example, } \\
\text { blaming or scapegoating. }\end{array}$ \\
\hline Multigenerational Transmission & $\begin{array}{l}\text { Particular issues or problems persist within the unit despite } \\
\text { leadership change. }\end{array}$ \\
\hline Nuclear Family Emotional System & $\begin{array}{l}\text { Patterns where individuals are functioning in parallel to others } \\
\text { within the 'family' (team/ unit) group. A situation where each } \\
\text { member of the group is impacted by the emotional atmosphere } \\
\text { within the group. This may result in blending of fusing of } \\
\text { individuals emotional selves. }\end{array}$ \\
\hline Sibling Position & $\begin{array}{l}\text { Ability to function is impacted by the individual's birth order and } \\
\text { number of other siblings. }\end{array}$ \\
\hline Triangling & $\begin{array}{l}\text { A configuration of three individuals, where the third person may } \\
\text { stabilise the relationship. The third person alleviates the stress } \\
\text { and tension that may exist between the initial two individuals. } \\
\text { Triangles may interlock resulting in either increased or decreased } \\
\text { anxiety within the system, depending upon the level of } \\
\text { differentiation of individuals involved. }\end{array}$ \\
\hline
\end{tabular}




\section{Application of Bowen Family Systems Theory to organisations}

Bowen Family Systems Theory has commonly been used to understand and assist in family process; however, it has also been applied to many other systems where there is potential for intense dysfunction or anxiety (Bowen Center, 2017). Triangling, emotional process and differentiation (Table 1) are the components of BFST most commonly applied to organisations (Bowen, 2012). In healthcare BFST has been most often applied to caring for patients (Leon \& Knapp, 2008; Miller \& Winstead-Fry, 1982) and their families (Duba Sauerheber, Nims, \& Carter, 2014; Haefner, 2014) rather than to the health service itself. However, organisational change consultants have supported the use of this theory to better understand and manage workplace operations, with a view to improving relationships and productivity (Kott, 2014).

\section{Anxiety in the workplace}

Anxiety is now more prevalent than ever in the workplace, due to the unprecedented rate of change and its multiple drivers such as technology, consumer expectations and rapid communication (Kott, 2014). This is important because employees' level of function is impacted by increasing anxiety within a work system (Kerr, 2012). Anxiety was first examined by Freud in 1925, with Klein (one of his pupils) continuing this work (Armstrong \& Rustin, 2015). Menzies (1960) drew on Kleinian perspectives to examine anxiety in the hospital setting, specifically among nurses. She was surprised at the level of anxiety nurses endured in the work situation that she suggested aroused strong feelings of 'pity, compassion, love, guilt and more anxiety' (Tutton \& Langstaff, 2015, p. 113). Nursing has evolved since then with changes in education, a more patient-centred approach, greater awareness of patient safety issues and performance targets (Tutton \& Langstaff, 2015); however, anxiety endures. 
Anxiety can be defined as a response to a threat, real or imagined (Kerr \& Bowen, 1988). According to Bowen's theory, differentiation and anxiety are processes explaining the functioning of a family or group, and BFST has been described as a 'map for managing' oneself within a system (Stern, 2012) during periods of high anxiety. Less differentiated and more anxious individuals are less able to adapt to stress (Kerr \& Bowen, 1988). Their objectivity can be maintained during periods of low anxiety but as anxiety increases, perspective is often lost. High levels of stress and anxiety have been found to impact cognitive abilities (Figley, 1995; Kott, 2014), potentially affecting problem solving and decision making - essential skills for critical care nurses to deliver safe and effective patient-centred nursing.

\section{Application of Bowen Family Systems Theory to a study of nursing in intensive care units}

A recent study using constructivist grounded theory methods discussed the complex relationships that exist in intensive care units (Jakimowicz et al., 2017b). Symptoms of anxiety depend on the level of stress and adaptive ability of the individual. Bowen's theory suggests that an understanding of the emotional processes within the workplace, such as intensive care units, takes some of the mystery from behaviours such as adaptiveness, reaction and response for which there may not appear logical explanation (Bowen, 1978). This awareness, informed by Bowen's theory, may also assist management groups and healthcare services in the support of their critical care teams without being caught up in the emotional process. Applying Bowen Family System Theory to an existing study of relationships in intensive care units is a way of thinking about the social processes and interactions already researched and may provide a distinctive understanding of how relationships work in this context. Application of BFST to the intensive care unit may also provide an effective approach to at least acknowledge, and perhaps alleviate, anxieties in this often chaotic environment (Kott, 2014). 


\section{Bowen Family Systems Theory critique}

Bowen's theory has its critics. Some view BFST as paternalistic, and a large body of work was undertaken by feminist scholars in response to these claims (Knudson-Martin, 1994). Nursing has a similar history, with medical dominance being aligned with paternalistic ideals (Germov, 2014). Supporting this point, Deborah Leupnitz, a well-known psychoanalytic couples therapist, suggested Bowenian therapy focused on the mother as a catalyst to symptom development in children (Leupnitz, 1988); others viewed overprotective mothers as being or causing un-differentiation. Some of these claims were clearly warranted, at least to some extent, as fathers were barely mentioned in Bowen's early work. Conversely, other scholars supported Bowen's work, hailing it as a theory that fitted well with feminist practice in assisting women to meet the challenge of remaining connected with their family (or group), but becoming differentiated (Knudson-Martin, 1994); having 'one's own voice' while still listening to others.

A further criticism of BFST has been the lack of importance placed on feelings, focusing on rational objectivity rather than the emotional processes so often used in therapeutic settings (Leupnitz, 1988). However, Knudson-Martin (1994) found the experience of exploring the 'tapestry' of the family system emotionally powerful. This is not unfamiliar to nurses. Historically, 'ways of nursing' were passed from nurse to nurse, based on precedents and traditional models (Wiechula, Conroy, \& McLiesh, 2016); a generational aspect also identified in healthcare. Although the rhetoric of evidence-based practice now predominates, the long tradition of experienced nurses 'showing the ropes' to junior nurses continues and is perpetuated in contemporary nursing (Wiechula et al., 2016). Evidence suggests that Bowen Family Systems Theory is a worthwhile form of therapy for individuals and groups, in families or any system, including organisations; empirical studies support the ideas and concepts of the 
model (Charles, 2001). The basic principles of BFST may be used to map a framework aimed at supporting critical care nurses, improving their wellbeing and the quality of care they provide.

\section{Data Sources}

This paper is based on published literature and texts that focus on Bowen's Family Systems Theory and findings of a constructivist study examining patient-centred nursing and compassion in Australian intensive care units (Jakimowicz et al., 2017a, 2017b).

\section{DISCUSSION}

This paper examines the potential to apply BFST to nursing in the intensive care unit. Studying a family, system or group rather than an individual is a key element underpinning Bowen's theory; consequently, this discussion focuses on the intensive care unit as a group (family), with critical care nurses the index individuals (family members).

\section{Recent study findings}

Findings from a constructivist study that examined compassion and patient-centred nursing in critical care are used to explain how BFST may be applied in intensive care units. The authors surveyed and interviewed critical care nurses from two Australian general adult intensive care units of one Sydney metropolitan and one regional hospital in Australia in 2016. Participants completed the Professional Quality of Life Survey V5 (PROQOL) (Hudnall Stamm, 2012) to determine their level and factors predictive of compassion satisfaction and compassion fatigue, and participated in individual interviews. Data from the quantitative phase were analysed using descriptive and inferential statistics (Jakimowicz et al., 2017a); qualitative analyses used Charmaz's constructivist methodology (Charmaz, 2014); themes were identified and theory developed (Jakimowicz et al., 2017b). 
Findings revealed early to mid-career critical care nurses at higher risk of fatigue than their more experienced, older colleagues; place of work differentiated and post graduate education pointed to higher levels of compassion satisfaction (Jakimowicz et al., 2017a). Critical care nurses' experience of compassion satisfaction and fatigue influenced their delivery of patientcentred critical care nursing. They were not satisfied when not able to deliver the care they believed their patients needed and deserved (Jakimowicz et al., 2017b). Nurses travelled backward and forward along a continuum between moments of compassion satisfaction and of compassion fatigue (Figure 1). Workplace culture and relationships were significant to critical care nurses' experiences of compassion satisfaction and fatigue, and in their delivery of effective patient-centred nursing (Jakimowicz et al., 2017b). An over-arching, core category of 'expectations' emerged, signifying tension between critical care nurses' biomedical, clinical skills and knowledge against compassionate, patient-centred nursing. Subthemes explaining this tension included 'life in the balance', 'passion and pressure', 'understanding and advocacy' and 'tenacity and fragility'. Critical care nurses' experiences of compassion and patient-centred nursing within their role and their working environment can be considered by mapping the study findings to components of BFST.

Figure 1 - Differentiation and Expectations 


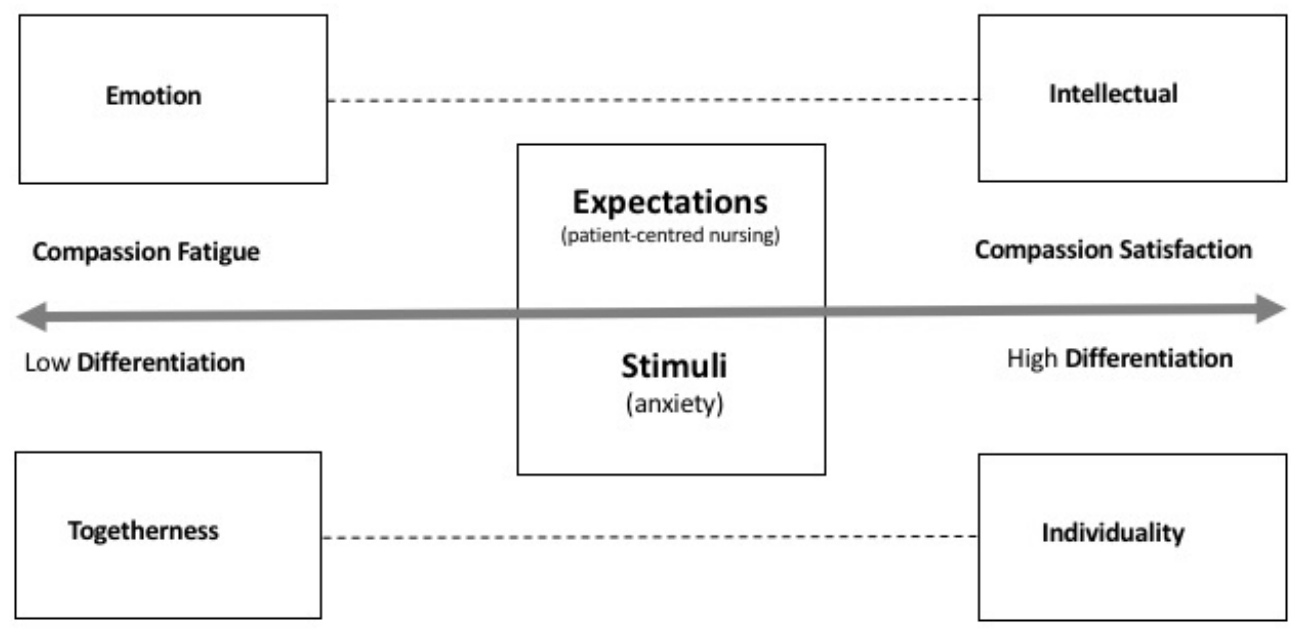

\section{Bowen Family Systems Theory in the intensive care unit}

The first step of BFST is to identify patterns of anxiety within a family or group (Bowen, 1978). The intensive care environment is prone to episodic anxiety due to patients' instability and the immediacy of their treatment requirements. As a consequence of this atmosphere critical care nurses participating in the study (Jakimowicz et al., 2017b) felt the effects of anxiety experienced by their colleagues and other health professionals working in this environment. Those interviewed explained feelings such as stress, distress, and wanting to escape; all symptoms of anxiety within a group. The high standards and expectations they took as their own, and those they perceived others had of them, added to the pressure. A theme, 'Life in the Balance', from the study results explains that balancing biomedical interventions with compassionate patient-centred nursing whilst managing multiple complex relationships was also anxiety provoking. These patterns of anxiety may impact compassion and patient-centred nursing in intensive care units.

Awareness of anxiety patterns and analysis of the emotional processes, including stimuli, reaction and response, can be facilitated through the general principles of Bowen's theory. 
Generally, symptoms of anxiety appear in the form of conflict, projection or illness (Bowen, 1978). In a family situation, there may be marital conflict, and a child or children may be the focus of projection and illness (Bowen, 1978). In the case of intensive care, medical roles (such as physicians) could be represented as 'masculine' and nursing roles as 'feminine' due to the medical dominance paradigm and historical paternalistic ideals (Germov, 2014). Conflict may occur between critical care nurses (Jakimowicz et al., 2017c), physicians (Aslakson et al., 2012; Hansen, Goodell, DeHaven, \& Smith, 2009), management teams (Jakimowicz et al., 2017b) and/or patients and families. Newcomers or newly graduated nurses, the less established members of the group, are often objects of projection from managers or more experienced critical care nurses during stressful periods. This was reflected in the constructivist study, where early and mid-career nurses were found to be at higher risk of fatigue (Jakimowicz et al., 2017b); as described in a theme 'stretching versus overstretching', they voiced their dismay and feelings of burden as their responsibilities within the unit increased. Some or all of these manifestations may be expressed, dependent upon the level of stress and the responses of other members of the group. These symptoms were apparent within the theme 'passion and pressure' within constructivist study findings (Jakimowicz et al., 2017b), where some nurses suffered compassion fatigue and others described feelings of anxiety and despair when they felt unsupported in providing biomedical care and patient-centred nursing simultaneously to their patient.

\section{Differentiation of Self}

The second step in BFST entails identifying and maintaining an individual position in an anxious setting, or an 'I' position' (Bowen, 2012). Differentiation of self is a concept that forms the basis of understanding maturity within a system. Put simply, it means the learned ability to think as an individual while remaining meaningfully connected to other members of a group 
(Kerr \& Bowen, 1988). It allows for healthy relationships where the individual may operate intellectually and emotionally independently without disengaging or distancing themselves (Bowen, 1978). While the importance of the group is acknowledged, the individual is able to endure rejection, criticism and conflict, being less dependent on others emotionally. Poor differentiation sees the individual requiring approval and acceptance from others for what they are thinking, doing and saying.

Differentiation, a cornerstone of BFST, may be applied to any work environment (Kott, 2014). Behaviours such as disengagement or bullying are symptoms of a system suffering anxiety (Kerr, 2012). Disengagement is a symptom of compassion fatigue; critical care nurses in the study experienced, on average, mid-range levels of compassion fatigue, with mid and early career nurses suffering more (Jakimowicz et al., 2017a). The individual work environment was an influence on their experience of compassion fatigue and satisfaction; this supports consideration of group-based ways of working, and the BFST focus on patterns that develop in groups as a way to diffuse anxiety.

Anxiety may impact individuals' levels of perspective and function (Bowen, 2012). Systemic rules, professional and societal expectations trigger anxiety in some individuals (Kerr, 2012), dependent upon their level of self-differentiation. According to study findings, critical care nurses perceived provision of patient-centred nursing as an expectation of the regulatory body and the management team (Jakimowicz et al., 2017b). They saw themselves as expected to act and work autonomously. Self-differentiation allows critical care nurses to be able to meet this expectation. Well differentiated critical care nurses are likely to be more self-confident of their abilities to meet perceived expectations. Study findings revealed that more experienced critical care nurses reported lower burnout and increased compassion satisfaction. This could have 
been due to their increasing differentiation as they became more confident in their ability to meet expectations and grew healthy relationships within the unit.

Individuals with post graduate education had greater compassion satisfaction (Jakimowicz et al., 2017a), which could align with higher differentiation leading to greater independence and more autonomous working within the group. Higher burnout was related to lower compassion satisfaction. Nurses described feelings of satisfaction when they felt respected, were able to provide effective compassionate patient-centred nursing, maintain professional boundaries, felt confident in their ability to meet their own, their patients' and the management team's expectations. These characteristics all point to greater differentiation, being able to separate thoughts from emotions in a positive and productive way. Greater compassion satisfaction may accompany lower burnout in more differentiated individuals, in an altogether less anxious group. Self-differentiation works to take the pressure off individuals as emotions about high self-expectations are taken out of the equation allowing a more logical and measured approach (Bowen, 1976). The end result may be critical care nurses who experience greater compassion toward their patients, while maintaining professional boundaries.

\section{Triangling}

'Triangling' describes the fluid equilibrium of a three-person configuration or system. Triangled relationships can be continuous or generational, meaning that individuals can move in and out of triangle relationships within a group (Kerr \& Bowen, 1988). During calm times a two-person relationship may be stable but become strained when the emotional environment becomes more stressful, requiring a third individual or entity to stabilise the situation. The level of comfort felt by each point of the triangle (or individual) is dependent upon the level of environmental stress. As anxiety levels increase, comfort levels may decrease and the emotional processes within a triangle relationship alter its characteristics (Kerr, 2012). The 
intensity of 'triangling' differs for each group and over time as individuals change and adapt.

Triangles may be a result of lack of differentiation, where emotional reactions are stronger than intellectual thinking, which may explain the variety of processes among different groups (Bowen, 2012). Triangles, or relationships, may become interlocked and spread when anxiety levels are high (Kerr, 2012) (Table 2).

Table 1 Triangling examples

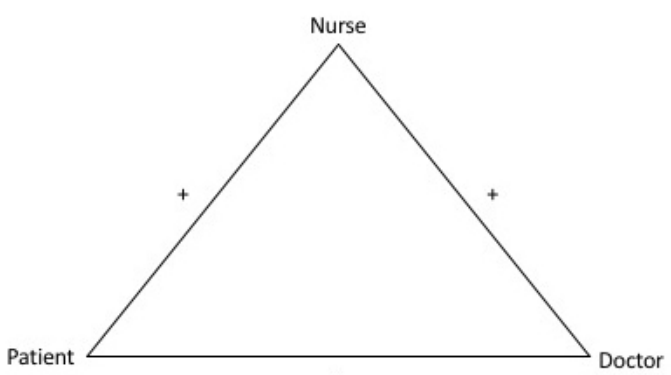

Patient-nurse-doctor

A nurse attempting to advocate for their patient's treatment plan is caught in a patient-nurse-doctor triangle. As critical care nurses spend a lot of time with their patient, they know their patients well. Moral distress may occur if continuing treatment for a patient with a poor prognosis. If the nurse reacts emotionally when disagreeing a treatment plan outlined by the doctor, the nurse appears to be 'taking the patients' side' emotionally. This triangle may meet a need in the nurse, but creates a situation of dismissal, endorsing medical dominance. A more differentiated nurse may use their biomedical knowledge to advocate for their patient in a more professional, less emotional manner.

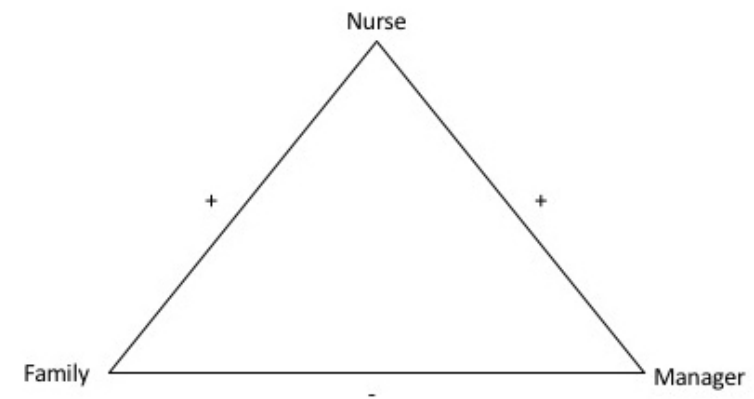

Nurse-family-manager

A critical care nurse caught in an intense situation with family members may actively recruit the involvement of a clinical manager. Conflict may still exist between the new players, so the nurse 
withdraws from the situation. The family may then attempt to draw the nurse back into the triangle to precipitate conflict between the nurse and clinical manager. When the anxiety decreases and the clinical manager withdraws, the family may then move closer to the nurse again, dismissing the clinical manager.

Interlocking Triangles

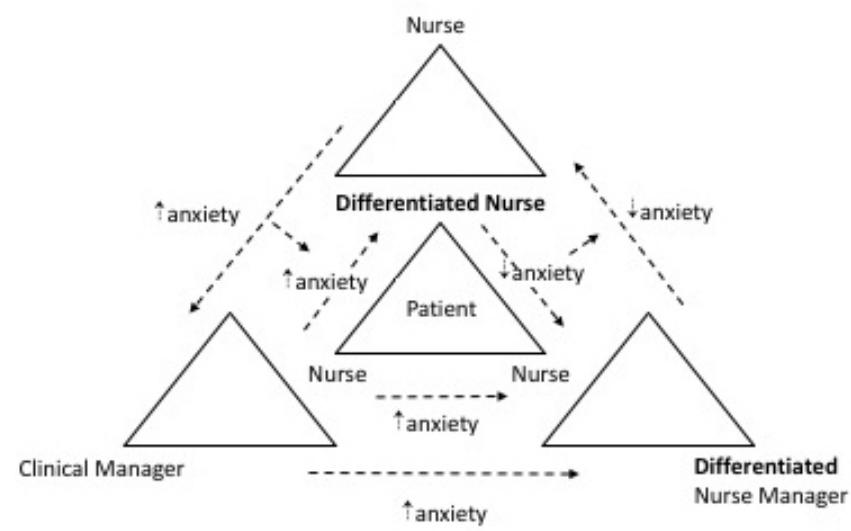

Nurse-clinical manager-Nurse Manager

Hospital administration set targets in-line with government policy (eg 4-hour Emergency Room rule). The clinical manager is accountable to the nurse manager and must enforce these targets. The critical care nurse may be pushed to meet these targets by the clinical manager. A well differentiated critical care nurse, while advocating for their patient must take an 'I' position (differentiated) in this situation, maintaining a calm stance, connected to their patient, without reacting emotionally. If less differentiated, anxiety may increase and a third or fourth person drawn in, forming an interlocking triangle. Dependent upon the level of differentiation within each individual the anxiety may increase if emotionally reactive, or decrease if responding in an intellectual manner. A well differentiated clinical manager would also take an 'I' position. Differentiation results in both critical care nurse and clinical manager finishing their shift without a negative response and the system, including the nurse.

Societal as well as professional expectations may be components of a triangled relationship. Meeting expectations played a large part in the constructivist study of critical care nurses' role (Jakimowicz et al., 2017b). The ability to develop a therapeutic relationship is expected of critical care nurses and an essential component of patient-centred nursing in any healthcare setting (Stein-Parbury, 2009), including critical care (Jakimowicz \& Perry, 2015). The theme of 'self' from the constructive study (Jakimowicz et al., 2017b) aligns well with this concept of BFST, where nurses have high self-expectations but also felt the pressure of meeting others' expectations (including colleagues, families and patients), often 'triangling' in the process. At times, this may be compromised in intensive care as a large number of patients are sedated, intubated or cognitively impaired (Jakimowicz et al., 2017c). However, critical care nurses 
become creative and often get to know their patient through developing close relationships with family members (Jakimowicz et al., 2017c).

\section{Emotional processes within intensive care}

Emotional process is the transmission of the parents' lack of differentiation to the child in a family situation (Kerr \& Bowen, 1988). This could align with a situation in which anxiety in the management team impacts bedside critical care nurses. 'Place of work' differentiated the compassion satisfaction and fatigue levels experienced by nurses, with the levels of anxiety within one unit compared to another perhaps responsible for the impact on critical care nurses' wellbeing, and possibly signifying differences in management team differentiation (Jakimowicz et al 2017a). Multi-generational transmission is a concept that may align with management styles within a workplace (Table 1). The different management styles in the two units where participants worked may have contributed to the predictive power of "place of work' for both compassion satisfaction and fatigue. In any group or system each individual is a participant; members of the management team, in particular, are not able to dismiss their part in the success or demise of the team (Stern, 2012). As anxiety builds at more senior levels it permeates through each member of the group. Relationships were important to participants of the study; they wanted healthy supportive connections including mentoring, practical and emotional support from their management team. Anxiety contributes to compassion fatigue, putting critical care nurses' wellbeing at risk, but from a systemic viewpoint no one individual is responsible; the issue resides within the group (Kerr \& Bowen, 1988).

The way individuals think and behave may be altered by the feelings and emotions experienced in the interplay of a two, three or four-person relationship (Bowen, 1978). According to Kerr and Bowen (1988), this process is regulated by 'individuality' or 'togetherness'. Critical care nurses in the study confirmed the importance of relationships with their colleagues; 
collaboration within the work environment could be dependent upon the individual teams. Each person within the group may be impacted by the emotional atmosphere, resulting in fused, blended, unhealthy or healthy relationships (Kerr \& Bowen, 1988).

Emotional reactivity is the response to environmental events. For example, anxiety within an environment such as an intensive care unit may invoke emotional reactions of varying degrees. Bowen proposes emotional reactivity may move along a continuum, aligning with more or less differentiation, ranging from hyper to hypo activity with one extreme being emotional paralysis (Kerr \& Bowen, 1988) (Figure 1). Bowen (1976) suggests that the lower the degree of differentiation, the more intense fusion within/between relationships, leading to more emotive than intellectual reactions to situations of increased anxiety.

\section{Conceptual framework}

Conceptual frameworks provide a means to explain and understand the world. In other words, a conceptual framework links existing knowledge with newly formed knowledge that has evolved from examination of specific concepts (Crookes \& Davies, 2004). The conceptual framework described in this paper extends the findings of the original study through a critical analysis and application of BFST to provide a new perspective of patient-centred nursing and compassion satisfaction. A diagram representing the conceptual framework is illustrated in Figure 2.

Figure 2 - Conceptual Framework 


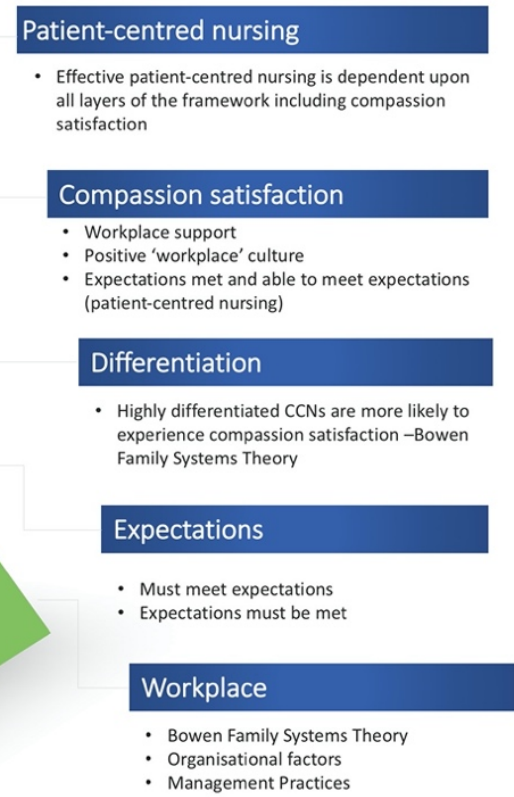

The framework comprises five levels, with each level drawn from results of the research and aligned with components of the BFST. This approach saw the 'parent theory' of BFST mapped to ideas and concepts derived by the constructivist study examining the complex relationships within the intensive care environment (Walker \& Avant, 2011) Concepts include workplace, expectations, differentiation, patient-centred nursing and compassion. Existing knowledge and new knowledge (BFST and examination of intensive care nursing) is threaded through the framework. Each level is dependent on the previous with workplace factors providing the foundation at the base of the pyramid. According to the critical care nurses, organisational factors and management practices provided the basis for both their successes and failures in fulfilling their role with satisfaction or fatigue (Jakimowicz et al., 2017b). Implementation of BFST in the workplace provides a foundation on which to build a positive workplace culture where individuals are empowered (Bowen, 2012).

The second layer of the pyramid represents the critical care nurses' expectations, of themselves and of others including their management team. The third level outlines differentiation (thinking intellectually rather than emotionally reacting to a situation or event), a concept 
seeing individual critical care nurses empowered in their role and contributing to their own success and satisfaction, as well as enhancing the workplace culture. The last two layers form a virtuous cycle, where delivering patient-centred nursing enhances compassion satisfaction and compassion satisfaction supports delivery of effective patient-centred nursing. This hierarchical figure signifies a pivotal point, with critical care nurses balancing movement backward and forward along a continuum between experiencing either compassion satisfaction or compassion fatigue. Each concept within this pyramid specifies a balancing point for critical care nurses' experiences of compassion satisfaction and fatigue.

\section{Implications for nursing}

Critical care nurses are key members of the intensive care unit and their wellbeing may be impacted by the anxiety levels that exist in the healthcare system or unit where they are working (Bowen, 2012). In turn, their capacity to provide compassionate patient-centred nursing may also be affected. Working with individual critical care nurses to improve anxiety levels within these groups may be important; however, given their strong affiliations with the system, such as an intensive care unit, working at a system level, at least initially, may be more powerful (Corey, 2008). Translation of BFST into critical care nursing requires a layered or stepped response supported by concepts within the framework. Maben and Bridges (2020) suggest strategies designed to support nurses should be aimed at an organisational and then team level before solely concentrating on individuals.

The conceptual framework is based on organisational factors and management practices; this is similar to BFST where the initial focus is on identifying patterns of anxiety within the family group. Preliminary strategies should take into account team cohesion; between colleagues (horizontally) and leaders (vertically) (Watson, 2020). This approach requires leaders and managers to be approachable, visible and transparent (Maben \& Bridges, 2020). Regular 
communication, where feedback is invited, builds relationships of trust and allows for identification of patterns of anxiety.

According to Bowen (2012), identification of anxiety within a system and assisting team members in greater differentiation are important factors in improving the workplace culture. The episodic and underlying causes of anxiety within an intensive care unit can be attributed to the acuity of patients, the essential immediacy of treatment, pressures around bed management and adherence to healthcare system guidelines and protocols (Jakimowicz et al., 2017b). Recognition of the emotional processes within the system is a fundamental step in improving workplace culture (Bowen, 2012).

Self-expectations and those of others sometimes can lead to high levels of anxiety (second layer of the conceptual framework). A healthy level of connectedness is an essential element in improved wellbeing and team cohesiveness (Watson, 2020). It is important for members of the critical care nursing team to respect each other's individuality, be inclusive and provide a space that is safe and welcoming where members of the team feel valued (Maben \& Bridges, 2020). An example of this is Schwartz Rounds or Team Time, which provide a forum where critical care and other health professionals come together to hear each other's stories; they benefit from 'normalising' their feelings, gaining a good understanding of expectations of others in their team (Maben et al., 2018).

The goal of BFST is to decrease blaming and reactivity and increase individual members' differentiation and self-responsibility. Therapy consists of three broad phases: 1) helping individuals identify patterns of behaviour/anxiety within the system; 2) assisting individuals to resist the pull of 'togetherness', remaining strong to themselves and not returning to old habits; 3) educating individuals in differentiating themselves from the system, increasing their selfresponsibility and decreasing anxiety. Bowen's theory is not focussed on technique; rather it 
provides a basis on which to structure therapy, dependent upon the system (Kerr \& Bowen, 1988).

Assisting critical care nurses to resist the pull of 'togetherness' will allow for development of greater differentiation. This is a progressive interplay, moving between autonomy (individuality) and connectedness (togetherness) (Kerr \& Bowen, 1988). Differentiation is a platform on which to grow and develop personally and professionally. Differentiation does not mean that critical care nurses are alone. An important implication of applying BFST to critical care nursing is finding the right balance to ensure individuality and connectedness. Peer to peer support has long been central to nurse wellbeing. This is still the case. It is important to acknowledge colleagues' feelings of anxiety at times and provide support by checking in, being open and approachable as well as listening (Maben \& Bridges, 2020).

Bowen conceptualises individual functioning as occurring along a continuum, from those least to most differentiated (Kerr \& Bowen, 1988). Similarly, the constructivist study found that critical care nurses moved backward and forward along a continuum between compassion satisfaction and fatigue (Figure 1) (Jakimowicz et al., 2017b). Self-differentiation aligns with how nurses experience compassion satisfaction and fatigue. Experienced at various times throughout a critical care nurse' professional life, some recover from the fatigue to recapture their compassion satisfaction (Coetzee \& Klopper, 2010), while others are less resilient. Better differentiated nurses may still suffer symptoms when stressed or at times of high anxiety, but they recover well. Less differentiated nurses may find it more difficult to recover; may leave the profession or become more unwell. It is important to note that resilience has been touted as an 'individual trait' (Traynor, 2018), however as discussed, organisational factors and workplace practices play an important role in supporting the occurrence of compassion satisfaction and patient-centred nursing. 


\section{Limitations}

Bowen Family Systems Theory was conceptualised in the 1960's and could be considered dated in dealing with organisational issues in contemporary healthcare. However, BFST has evolved over time, has been easily adapted by organisational change consultants across many settings and successfully integrated into those settings to improve productivity and staff wellbeing (Kerr, 2012; Kott, 2014).

\section{CONCLUSION}

Application of BFST to the intensive care unit may be used as a tool to improve health professional wellbeing, workforce retention and patient satisfaction. This paper has made plain the importance of having a clear understanding of the anxiety that exists within the intensive care unit as a system, so that individual members of the system, such as critical care nurses, can be supported appropriately. Anxiety levels within organisations impact the wellbeing and functioning of members within that system (Bowen, 1976). Critical care nurses rely on each other and their management team for support to ensure they meet the expectations of their role. Where organisational factors and management approaches fall short in supporting critical care nurses to meet their expectations BFST can offer a perspective on the social processes within the unit that impact nurse wellbeing and quality of care. Every system, whether a family, business or healthcare unit, functions differently; assessment and intervention to improve workplace culture and relationships should be based on the unique characteristics and needs of the group. BFST offers a unique approach to address the systems of care issues that undermine patient-centred nursing, leading to compassion fatigue in critical care nursing.

\section{References}

Abbey, M., Chaboyer, W., \& Mitchell, M. (2012). Understanding the work of intensive care nurses: a time and motion study. Australian Critical Care, 25, 13-22. 
Armstrong, D., \& Rustin, M. (2015). Introduction: revisiting the paradigm. In D. Armstrong \& M. Rustin (Eds.), Social defences against anxiety: exploratations in a paradigm (pp. 123). London, UK: Karnac Books.

Aslakson, R. A., Wyskiel, R., Thornton, I., Copley, C., Shaffer, D., Zyra, M., . . . Pronovost, P. J. (2012). Nurse-Perceived Barriers to Effective Communication Regarding Prognosis and Optimal End-of-Life Care for Surgical ICU Patients: A Qualitative Exploration. Journal of Palliative Medicine, 15(8), 910-915. doi:10.1089/jpm.2011.0481

Beckstrand, R., Hadley, K., Luthy, K., \& Macintosh, J. (2017). Critical care nurses' suggestions to improve end-of-life care obstacles: minimal change over 17 Years. Dimensions of Critical Care Nursing, 36(4), 264-270.

Bowen Center. (2017). The Bowen Center for the Study of the Family. Retrieved from https://thebowencenter.org

Bowen, M. (1976). Theory in the practice of psychotherapy. In P. Guerin (Ed.), Family therapy. New York, NY: Gardner Press Inc.

Bowen, M. (1978). Family therapy in clinical practice. New York, NY: Jasonn Aronson.

Bowen, M. (2012). Introduction. In R. Sagar \& K. Wiseman (Eds.), Understanding organisations: applications of Bowen Family Systems Theory (pp. vii-xii). Washington DC, USA: Fifth Printing.

Chambers, M. (2009). Nothing is as practical as a good theory: Bowen Theory and the workplace - a personal application. Australian and New Zealand Journal of Family Therapy, 30(4), 235-246.

Charles, R. (2001). Is There Any Empirical Support for Bowen's Concepts of Differentiation of Self, Triangulation, and Fusion? American Journal of Family Therapy, 29(4), 279292. doi:10.1080/01926180152588707

Charmaz, K. (2014). Constructing grounded theory. CA, USA: Sage Publications.

Coetzee, S., \& Klopper, H. (2010). Compassion fatigue within nursing practice: a concept analysis. Nursing \& Health Sciences, 12(2), 235-243.

Corey, G. (2008). Theory and practice of group counselling (7th ed.). Belmont, CA: Thomson Higher Education.

Crookes, P., \& Davies, S. (2004). Research into practice: essential skills for reading and applying research in nursing and health care. Edinburgh: Bailliere Tindall.

Duba Sauerheber, J., Nims, D., \& Carter, D. (2014). Counseling Muslim couples from a Bowen Family Systems perspective. The Family Journal: Counseling and Therapy for Couples and Families, 22(2), 231-239.

Epp, K. (2012). Burnout in critical care nurses: a literature review. Dynamics, 23(4), 25-31. Retrieved 
http://search.ebscohost.com/login.aspx?direct=true \&db=c8h\&AN=2011768955\&site $=$ ehost-live

Figley, C. (1995). Compassion Fatigue: Coping with Secondary Traumatic Stress Disorder in Those Who Treat the Traumatized. New York, NY: Brunner/Mazel.

Francis, R. (2013). Report of the Mid Staffordshire NHS Foundation Trust Public Inquiry: Executive Summary. London, UK: The Stationery Office Limited

Germov, J. (2014). Challenges to medical dominance. In J. Germov (Ed.), Second opinion: an introduction to health sociology (pp. 388-409). South Melbourne, VIC: Oxford University Press.

Haefner, J. (2014). An application of Bowen Family System Theory. Issues in Mental Health Nursing, 35(11), 835-841.

Hansen, L., Goodell, T. T., DeHaven, J., \& Smith, M. (2009). Nurses' perceptions of end-oflife care after multiple interventions for improvement. American Journal of Critical Care, 18(3), 263-271. doi:10.4037/ajcc2009727

Hudnall Stamm, B. (2012). Professional quality of life: compassion satisfaction and fatigue version 5 (ProQOL).

Jakimowicz, S., \& Perry, L. (2015). A concept analysis of patient-centred nursing in the intensive care unit. Journal of Advanced Nursing, 71(7), 1499-1517. doi:10.1111/jan.12644

Jakimowicz, S., Perry, L., \& Lewis, J. (2017a). Compassion satisfaction and fatigue: a crosssectional survey of Australian intensive care nurses. Australian Critical Care, 30(2), 127 (online).

Jakimowicz, S., Perry, L., \& Lewis, J. (2017b). Insights on compassion and patient-centred nursing in intensive care: a constructivist grounded theory. Journal of Clinical Nursing, 27(7), 1599-1611.

Jakimowicz, S., Perry, L., \& Lewis, J. (2017c). An integrative review of supports, facilitators and barriers to patient-centred nursing in the intensive care unit. Journal of Clinical Nursing, 26(23-24), 4153-4171. doi:10.1111/jocn.13957

Jones, J., Winch, S., Strube, P., Mitchell, M., \& Henderson, A. (2016). Delivering compassionate care in intensive care units: nurses' perceptions of enablers and barriers. Journal of Advanced Nursing, 72(12), 3137-3146. doi:10.1111/jan.13064

Karanikola, M. N. K., Albarran, J. W., Drigo, E., Giannakopoulou, M., Kalafati, M., Mpouzika, M., . . . Papathanassoglou, E. D. E. (2014). Moral distress, autonomy and nursephysician collaboration among intensive care unit nurses in Italy. Journal of Nursing Management, 22(4), 472-484. doi:10.1111/jonm.12046

Kelleher, S. (2006). Providing patient-centred care in an intensive care unit. Nursing Standard, 21(13), 35-40. Retrieved from https://www.lib.uts.edu.au/goto?url=http://search.ebscohost.com/login.aspx?direct=tr ue $\& \mathrm{db}=\mathrm{a} 9 \mathrm{~h} \& \mathrm{AN}=23652207 \&$ site $=$ ehost-live 
Kerr, M. (2000). One family's story: a primer on Bowen theory. Retrieved from https://thebowencenter.org/theory/

Kerr, M. (2012). Application of Family Systems Theory to a work system. In R. Sagar \& K. Wiseman (Eds.), Understanding organisations: applications of Bowen Family Systems Theory (pp. 121-131). Washington DC, USA: Fifth Printing.

Kerr, M., \& Bowen, M. (1988). Family evaluation: the role of the family as an emotional unit that governs individual behaviour and development. Ontario, Canada: Penguin Books.

Knudson-Martin, C. (1994). The female voice: applications to Bowen's Family Systems Theory. Journal of Marital and Family Therapy, 20(1), 35-46. doi:10.1111/j.17520606.1994.tb01009.x

Kott, K. (2014). Applying Bowen Theory to Work Systems. OD Practitioner, 46(3), 76-82. Retrieved from http://ezproxy.uow.edu.au/login?url=http://search.ebscohost.com/login.aspx?direct=tr $\mathrm{ue} \& \mathrm{db}=\mathrm{bth} \& \mathrm{AN}=97081024 \&$ site $=$ eds-live

Leon , A., \& Knapp, S. (2008). Involving family systems in critical care nursing. Dimensions of Critical Care Nursing, 27(6), 255-262.

Leupnitz, D. (1988). The family interpreted: psychoanalysis, feminism and family therapy. New York, NY: Basic Books.

Maben, J., \& Bridges, J. (2020). Covid-19: Supporting nurses' psychological and mental health. Journal of Clinical Nursing, n/a(n/a). doi:10.1111/jocn.15307

Maben, J., Taylor, C., Dawson, J., Leamy, M., McCarthy, I., Reynolds, E., . . Foot, C. (2018). A realist informed mixed-methods evaluation of Schwartz Center Rounds ${ }^{\mathrm{TM}}$ in England. Retrieved from

McCormack, B., \& McCance, T. (2016). The person-centred practice framework. In B. McCormack \& T. McCance (Eds.), Person-centred practice in nursing and health care (pp. 36-66). West Sussex, UK: John Wiley \& Sons Ltd.

Menzies, I. (1960). A case-study in the functioning of social systems as a defence against anxiety: a report on a study of the nursing service of a general hospital. Human Relations, 13, 95-121.

Miller, S., \& Winstead-Fry, P. (1982). Family systems theory in nursing practice. Reston, Virginia: Reston Publishing Company.

Mitchell, M. L., Coyer, F., Kean, S., Stone, R., Murfield, J., \& Dwan, T. (2016). Patient, family-centred care interventions within the adult ICU setting: An integrative review. Australian Critical Care, 29(4), 179-193. doi:https://doi.org/10.1016/j.aucc.2016.08.002

Sagar, R., \& Wiseman, K. (Eds.). (1982). Understanding organizations: applications of Bowen family systems theory. Washington: Georgetown University Family centre. 
Stein-Parbury, J. (2009). Patient and person: interpersonal skills in nursing (4th ed.). Chatswood, NSW: Churchill Livingstone Elsevier.

Stern, D. (2012). Application of Bowen Family Systems Theory in the motion picture industry. In R. Sagar \& K. Wiseman (Eds.), Understanding organisations: applications of Bowen Family Systems Theory (pp. 95-100). Washington DC, USA: Fifth Printing.

Traynor, M. (2018). Guest editorial: What's wrong with resilience? Journal of Research in Nursing, 23(10), 5-8.

Tutton, E., \& Langstaff, D. (2015). Reflections on Isabel Menzies Lyth in the light of developments in nursing care. In D. Armstrong \& M. Rustin (Eds.), Social defences against anxiety: exploratations in a paradigm (pp. 111-123). London, UK: Karnac Books.

Walker, L., \& Avant, K. (2011). Strategies for theory construction in nursing (5th ed.). Upper Saddle River, NJ: Prentice Hall.

Watson, P. (2020). Caring for yourself and others during the COVID-19 pandemic: managing healthcare workers' stress. Compassion in Action Webinar Series. Retrieved from https://www.theschwartzcenter.org/media/Schwartz-Center-Watson-SFA-FinalPDF.pdf

WHO. (2014). WHO global strategy on people-centred and integrated health services. Retrieved from http://www.who.int/servicedeliverysafety/areas/people-centredcare/en/

Wiechula, R., Conroy, T., \& McLiesh, P. (2016). Evidence-based practice/knowledge transationa: a practical guide. In E. Chang \& J. Daly (Eds.), Transitions in nursing: preparing for professional practice (5th ed.). Chatswood, NSW: Elsevier. 